\title{
Mass estimation and discrimination during brief periods of zero gravity
}

\author{
HELEN E. ROSS \\ University of Stirling, Stirling, Scotland \\ and \\ MILLARD F. RESCHKE \\ Neuroscience and Behavior Laboratory, NASA, Johnson Space Center, Houston, Texas
}

\begin{abstract}
Under zero gravity, the gravitational cues to mass are removed, but the inertial cues remain. A sensation of heaviness is generated if objects are shaken, and hence given a changing acceleration. A magnitude estimation experiment was conducted during the 0-G phase of parabolic flight and on the ground, and the results suggested that objects felt lighter under $0 \mathrm{G}$ than under $1 \mathrm{G}$. Mass discrimination was also measured in flight, and yielded Weber fractions of 18 under $0 \mathrm{G}, .16$ under $1.8 \mathrm{G}$, and .09 under $1 \mathrm{G}$. Poor performance under microgravity and macrogravity was probably due mainly to lack of time for adaptation to changed $G$ levels. It is predicted that discrimination should improve during the course of prolonged spaceflight, and that there should be an aftereffect of poor discrimination on return to earth.
\end{abstract}

When we handle objects, we gain an impression of their mass. Normally, this activity is described as "weight perception" and the accompanying sensation is described as "heaviness." It does not matter what name is given to a sensation, but it is perhaps misleading to suggest that heaviness corresponds to the physical property of weight. Physicists disagree as to the definition of weight, although many would consider it to be the force exerted on a mass in the earth's gravitational field at the earth's surface. Mass, however, has an agreed definition, being the ratio of force to acceleration. Weight changes with the gravitational force, but mass remains constant. It is possible to compare the mass of two objects by comparing the force required to produce the same acceleration, or the difference in acceleration produced by imparting the same force. This remains true whether the objects are on earth or under weightless conditions. If the sensation of heaviness corresponds to weight, objects should feel totally weightless under zero gravity $(0 \mathrm{G})$; but if heaviness actually corresponds to mass, the objects should feel as heavy as under normal conditions on earth. If heaviness is a compromise between these two extremes, objects

This work was supported by a grant to the first author from the Medical Research Council. Part of the research was conducted while she was based at the DFVLR Institut für Flugmedizin, Bonn, supported by fellowships from the European Space Agency and the Royal Society. The mass-discrimination apparatus was constructed by the University of Stirling, RAE (Farnborough), and ESTEC (Noordwijk). We should like to thank J. Conkin for assistance with Experiment 1 and A. J. Benson, O. K. Garriott, and C. H. Oman for comments on a first draft of this paper. Correspondence may be directed to the first author at Department of Psychology, University of Stirling, Stirling FK9 4LA, Scotland. should feel lighter under $0 \mathrm{G}$ than under $1 \mathrm{G}$, but not totally weightless. Evidence for a compromise between weight and mass has been noted when the observer and the objects lose weight through immersion in water (Ross, Rejman, \& Lennie, 1972) or gain weight in the human centrifuge (Ross, 1981b). It would not be surprising if a similar compromise occurred under microgravity.

Despite a century and a half of research on lifted weights, we are still remarkably ignorant about which aspects of the physical stimulus are picked up by the sensory system, and which sensory mechanisms are involved in their analysis. Some experiments have been concerned with "apparent weight," and others with the ability to discriminate differences in weight or mass. The relation between sensation and discrimination is far from clear in any sense modality: different neural mechanisms or pathways may be used for the two abilities. We shall therefore discuss heaviness estimates and discrimination separately in the introductions to two different experiments.

\section{EXPERIMENT 1: HEAVINESS ESTIMATES}

It is generally found that there is a tendency towards mass constancy, despite variation in the effort required to lift an object. This has been noted despite the use of different parts of the body, or of variation in arm weight (Fischel, 1926), or of variation of the distance of an object along a lever (Torrey, 1963/ 1964). Constancy is good when the weight of the object is unchanged, and the variation lies in the manner of lifting. It is less good when the effective weight 
of the object changes, as for example underwater (Ross et al., 1972) or in the human centrifuge (Ross, $1981 \mathrm{~b}$ ). In such cases, the weight of the subject's arm also changes, and it is likely that this provides a cue to the change of weight in the object. For him to show any constancy, it is essential that the subject have some cues as to the nature of the transformation.

Constancy is poor in cases in which the objects are hidden from sight and subjects are forced to rely on the sense of effort or other cues. The sense of effort increases in cases of muscular fatigue or partial paralysis (Gandevia \& McCloskey, 1977; McCloskey, Ebeling, \& Goodwin, 1974); this sense appears to depend more on the efferent command signals to the muscles than on the afferent signals from muscle and joint receptors, although both are implicated (Ross \& Bischof, 1981).

It is clear that changes in expected effort, or in expected sensory input, are taken into account when estimating mass. These changes in expectation probably mediate mass constancy and adaptation in normal circumstances. In abnormal circumstances, they mediate illusions such as the size-weight illusion (Ross, 1969).

Apparent heaviness tends to decrease with a loss of sensitivity. Weber $(1834 / 1978)$ claimed that weights felt lighter when placed on those parts of the body that had poorer tactile sensitivity, but he referred only to passive pressure perception, not active lifting. Preliminary observations suggest that the same is true when objects are lifted by hands made insensitive through cold or pressure blocking.

What might be expected to happen when mass is perceived through inertial cues rather than through weight cues? Normally, during active lifting on earth, both cues operate. However, we usually move objects at much lower accelerations than the acceleration of gravity $\left(9.81 \mathrm{~m} / \mathrm{sec}^{2}\right)$, so the weight cues predominate. When the weight cues are removed, only the inertial cues remain, and the inertial forces are likely to feel small in comparison with weight. However, we normally take into account the rate at which we accelerate objects when judging weight or mass on earth, and there is no reason why we should not learn to do the same when judging mass under weightless conditions. Some opportunities for learning about inertial forces do exist on earth: for example, when pushing objects over a smooth surface, as in curling, ice hockey, or shove-ha'p'ny; when pushing objects suspended by a pendulum or on an airbearing table; or when manipulating neutrally buoyant objects underwater. We might expect subjects to show perceptual learning in these conditions, even if they exhibit little mass constancy on the first occasion.

An experiment on mass perception through inertial cues was performed by Crawford and Kama (Note 1). They required their subjects to make absolute esti- mates of the mass of objects after pushing them over an air-bearing table. The objects weighed 2,6 , and $10 \mathrm{lb}(.91,2.72$, and $4.54 \mathrm{~kg})$. The subjects estimated their mass in pounds, both with normal lifting and when pushed over the frictionless surface. The ratios of "weight" to "mass" estimates were $1.16,1.52$, and 1.95 , respectively, suggesting an increasing underestimation of mass as judged by inertial cues rather than weight cues. However, it is not entirely clear whether the underestimation was due to the loss of the normal pressure cues to weight or to the loss of the normal method of lifting. The experiment does not simulate the conditions of weightlessness for at least two reasons: the subject's arm remains weighted, and the arm must not be raised in the normal manner. It is possible that greater mass constancy would be shown under true weightlessness, although there appears to be no hard evidence on the subject. Anecdotal reports by astronauts and cosmonauts suggest that both other objects and their own bodies feel "too light" in orbit and "too heavy" on return to earth. Salyut cosmonauts estimated objects as feeling 2-3 times their normal weights immediately postflight, although the effect dissipated rapidly (Gazenko, Genin, \& Egerov, Note 2). This makes it likely that crewmembers adapt to the loss of weight in orbit, and experience an aftereffect of heaviness on return to earth.

Parabolic flight provides a useful opportunity for investigation of the effects of microgravity. Periods of up to $25 \mathrm{sec}$ of approximate $0 \mathrm{G}$ can be produced during each parabola, although this is preceded by about $20 \mathrm{sec}$ of $1.8 \mathrm{G}$ (where $\mathrm{G}$ is the acceleration of gravity and equals $\left.9.81 \mathrm{~m} / \mathrm{sec}^{2}\right)$. We made use of the $0-\mathrm{G}$ periods to investigate apparent heaviness.

\section{Method}

Twenty subjects were used, 13 men and 7 women. They were scientists, technicians, or secretaries working at the Johnson Space Center, with an age range of 18 to 30 years (mean, 25 years). Eighteen were right-handed and two left-handed. Only four had had previous experience of parabolic flight. Two additional male subjects, who failed to complete the experiment due to nausea, were dropped from the sample.

The subjects were tested on the ground at Ellington Air Force Base during the hour before and the hour after the flight and also during the $0-\mathrm{G}$ phase of parabolic flight. The flight tests were conducted in NASA's KC-135 aircraft, adapted for parabolic flight. The aircraft normally flies $\mathbf{4 0}$ parabolas per flight. The details of the flight pattern for an ideal parabola are shown in Figure 1. In practice, the acceleration during the pull out was usually less than during the pull up. A typical acceleration profile is shown in Figure 2.

The apparatus consisted of four containers with masses of 100 , 200,300 , and $400 \mathrm{~g}$. They were transparent plastic urine containers, lined with yellow paper and filled with lead shot and cotton wool. They had orange screw-on caps and cone-shaped ends. The height of the cylinders was $100 \mathrm{~mm}$ for the main body and $15 \mathrm{~mm}$ for the cone; the diameter was $30 \mathrm{~mm}$ for the main body and $35 \mathrm{~mm}$ for the cap. The containers rested in holes in a polystyrene transport box.

For the ground tests, the subject and the experimenter sat opposite to each other at a table. The subject was blindfolded and used 


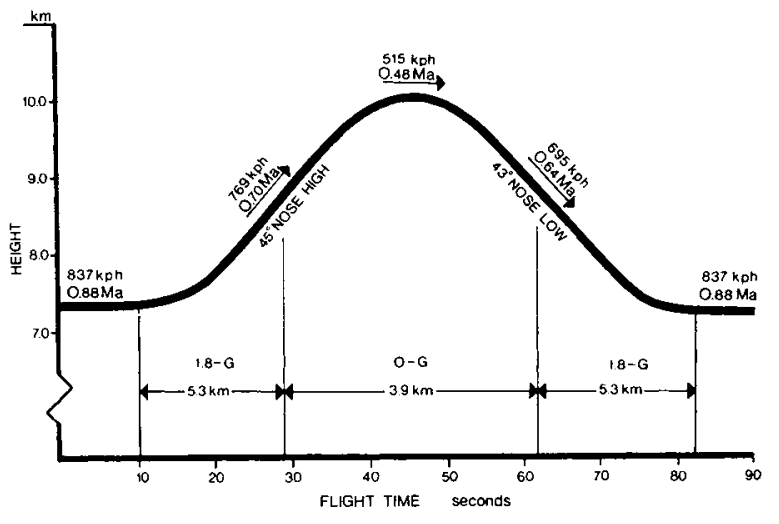

Figure 1. Flight pattern for an ideal parabola.

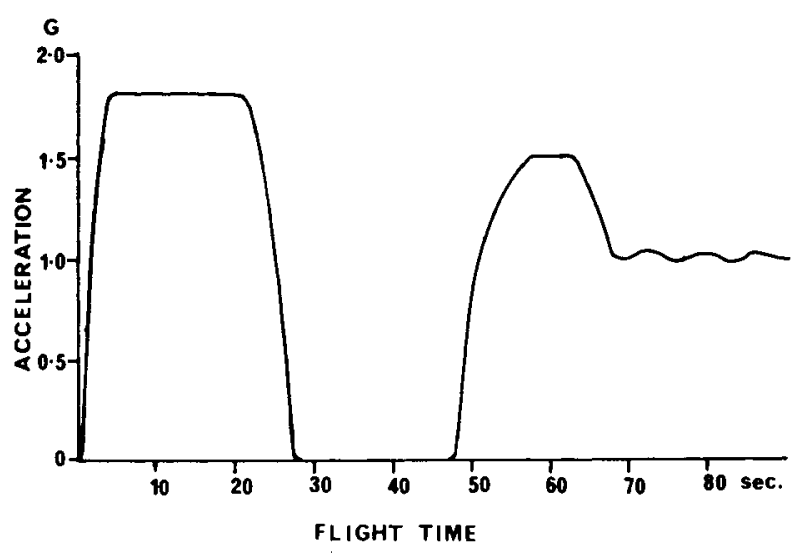

Figure 2. Typical variation in G level during a parabola.

his dominant hand for the test. The experimenter put the $100-\mathrm{g}$ container in the subject's hand and instructed him to give it a value of 100 units. The subject shook it gently in the hand two or three times and then gave it back to the experimenter. The experimenter then gave him all four containers in a random order, and the subject estimated the heaviness of each in relation to his memory of the standard.

For the flight tests, both the subject and the experimenter were seated and strapped into chairs beside each other. All subjects were blindfolded, except for four who wore red goggles as part of another experiment: they looked straight ahead and could not see which containers the experimenter was handling. The experimenter handed the 100-g standard to the subject just before the start of a parabola. The subject held the standard passively and then shook it at the beginning of the 0-G phase. He then returned it to the experimenter, who handed him all four containers in a random order. The subject made estimates as he had on the ground, performing as rapidly as possible so as to complete all the judgments within the $20-\sec 0-\mathrm{G}$ phase. All tests were conducted during the 4th-20th parabolas, during 4 days of flights. The majority of subjects were seated and blindfolded prior to the test, but two of the four with red goggles had moved around and participated in vestibular experiments beforehand.

\section{Results and Discussion}

The mean, median, and standard deviation of the magnitude estimates under the three conditions are shown in Table 1. The standard deviation increased with the mean, as is normal. An ANOVA revealed highly significant effects due to mass $[F(3,57)=29.2$, $\mathrm{p}<.0001]$ and test conditions $[\mathrm{F}(2,38)=12.9, \mathrm{p}=$ $.0001]$ and a significant interaction between the two $[F(6,114)=4.0, p=.0013]$. The $0-G$ estimates were significantly lower than the mean of the two ground tests $[F(1,38)=24.48, p<.0001]$, but the two ground tests did not differ significantly from each other $[F(1,38)=1.29]$.

The median estimates appear to increase approximately linearly with mass over the range tested (see Figure 3). Because of the small mass range and relatively small number of judgments, no attempt at curvefitting was made. Magnitude estimates normally yield a power function (Stevens \& Galanter, 1957), but when the range is small a linear relation is common (Poulton, 1979). Approximately linear estimates have been found in other weight estimation experiments (Ross et al., 1972).

The main result of the experiment is that the $0-\mathrm{G}$ estimates were lower than the 1-G estimates. Curiously, this was true even for the $100-\mathrm{g}$ stimulus, which might have been expected to have the same value under all conditions. The ratios of the median $1-G$ to median $0-G$ estimates ranged from 1.5 to 1.2 , but there was no systematic increase with mass as had been found by Crawford and Kama (Note 1). The ratios were also slightly lower than those of Crawford and Kama, which ranged from 1.2 to 2.0 . The results should not be taken to imply that objects necessarily feel about three-quarters as heavy in weightless conditions as in 1-G conditions, since the standard was assigned the same value under both conditions: If the standard were to change in apparent weight in the same proportion as the comparison stimuli, no difference between the conditions should manifest itself. Clearly, this was not the case. The results are hard to interpret,

Table 1

Magnitude Estimates in Relation to a $100 \mathrm{~g}$ Standard With a Nominal Value of 100

\begin{tabular}{lcccc}
\hline & \multicolumn{4}{c}{ Comparison Stimulus (in Grams) } \\
\cline { 2 - 5 } & 100 & 200 & 300 & 400 \\
\hline \multirow{5}{*}{ Pretest (1-G) } \\
Mean & 85.0 & 210.5 & 308.8 & 431.3 \\
Median & 75.0 & 200.0 & 250.0 & 350.0 \\
SD & 41.7 & 95.5 & 194.2 & 272.8 \\
& \multicolumn{5}{c}{} \\
Mean & 71.3 & 156.3 & 193.5 & 360.3 \\
Median & 50.0 & 150.0 & 200.0 & 325.0 \\
SD & 42.4 & 94.9 & 159.2 & 240.6 \\
& & Plight & $(0-\mathrm{G})$ \\
Mean & 73.0 & 227.5 & 336.3 & 476.3 \\
Median & 75.0 & 200.0 & 300.0 & 410.0 \\
SD & 27.6 & 103.2 & 222.1 & 273.8 \\
\hline
\end{tabular}

Note-Ratio of $1-G$ to $0-G$ medians $=1.50,1.33,1.38$, and 1.17 for 100-, 200-, 300-, and 400-g comparison stimuli, respectively. 


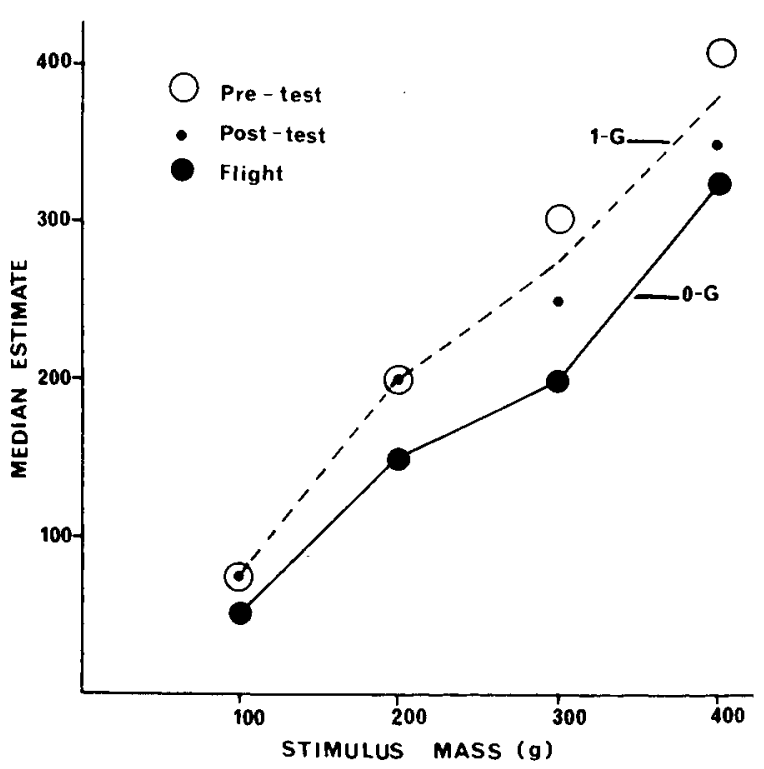

Figure 3. Median magnitude estimates under $0 \mathrm{G}$ (flight) and 1 G (ground).

because the standard was presented during the $0-\mathrm{G}$ phase. It remains obscure, then, as to whether the reduction in apparent heaviness is related to the facts of physics, to neurophysiological processes, or to judgmental biases in scaling procedures (Poulton, 1979).

A variation in the test procedure was carried out in an attempt to clarify the issue. The standard was presented only under 1-G conditions, and subjects made judgments under $0 \mathrm{G}$ in relation to their memory of the standard at $1 \mathrm{G}$. Seven subjects from Experiment 2 were tested in flight during one or two parabolas following their discrimination test. They were presented with a 50-g ball during the $1-\mathrm{G}$ interparabola phase and were instructed to assign it a value of 100 units. They were then given other $50-\mathrm{g}$ balls during the following $1.8-\mathrm{G}, 0-\mathrm{G}$, and $1-\mathrm{G}$ periods. Subjects judged two balls under each condition. They were not told that all the balls presented had the same mass. The median magnitude estimates were 150 $(1.8 \mathrm{G}), 60(0 \mathrm{G})$, and $120(1 \mathrm{G})$. The $1-\mathrm{G}$ estimates were too heavy in relation to the standard, although it is not clear whether this was a shift in memory or a perceptual aftereffect. The $0-\mathrm{G}$ estimates were clearly lighter than the 1-G estimates, by a factor of about 2 . Understandably, the ratio was larger than in the previous experiment, in which the standard was held throughout the early phase of the parabola. It is interesting to note that the ratio of $1.8-\mathrm{G}$ to $1-\mathrm{G}$ estimates was 1.25 rather than 1.8 . This may represent a tendency toward mass constancy, similar to the effect found under macrogravity in the human centrifuge (Ross, 1981b).

There can be little doubt that the subjects felt objects to be too light during the $0-\mathrm{G}$ phase of parabolic flight. Nevertheless, it should not be assumed that objects will always feel too light under weightless conditions. The subjects were mainly inexperienced at parabolic flight and had little opportunity to adapt to the brief periods of microgravity interspersed with periods of macrogravity. It is probable that subjects do adapt during prolonged weightlessness in spaceflight, with the result that their own bodies and other objects come to have almost the same apparent heaviness as on earth. It is also possible that subjects may adapt a little during repeated parabolas. It is interesting to note that, in the main experiment, the posttest estimates were slightly greater than the pretest estimates. Although the difference was not significant, the trend was in the direction expected for an aftereffect. If it had been possible to test subjects immediately after the last parabola (instead of 1 to $2 \mathrm{~h}$ later), it is possible that a significant aftereffect would have been obtained.

\section{EXPERIMENT 2: MASS DISCRIMINATION}

Mass discrimination and apparent heaviness are indirectly related to one another, insofar as factors that cause a change in one may also cause a change in the other, although not always in the same direction. Two general rules emerge: (1) Discrimination improves with an increase in the sources of sensory information, or in the sensitivity of those sources; apparent heaviness also tends to increase under these circumstances. (2) Discrimination is optimal when the subject is well adapted to his current effective body weight and to the range of effective weights that he is comparing, and it deteriorates when he is adapted to inappropriately heavy or light conditions. When a subject is preadapted to a heavier range or a heavier body weight, a given test object feels "too light," and when he is preadapted to lighter conditions, it feels "too heavy."

The evidence for these statements is reviewed in greater detail by Ross (1981b). The first rule is fairly obvious. Discrimination thresholds may be raised by a factor of up to 2 through loss of the normal method of lifting, as in static rather than active discrimination (Holway \& Hurvich, 1937; Weber, 1834/1978). Thresholds are also raised during active lifting when the hands are cold rather than warm (Synodinos \& Ross, 1979) or when the wrist, rather than the shoulder, is used as a fulcrum (Holway \& Hurvich, 1937; Oberlin, 1936). A study of particular relevance to this investigation was conducted by Rees and Copeland (Note 3): they compared mass discrimination when the objects were pushed over an air-bearing table and when they were lifted normally. They found that thresholds were about twice as large with inertial cues alone as they were with weight cues. However, it is not clear how much of the increase was due to loss of weight cues 
and how much to loss of the normal method of lifting.

The second rule is perhaps less obvious, but there are many examples of the deleterious effect of maladaptation. The Weber fraction for mass discrimination increases when arm weight is suddenly increased as a result of the addition of a weighted cuff to the forearm (Gregory \& Ross, 1967) or acceleration in a human centrifuge (Ross, 1981b), or when arm weight is suddenly decreased as a result of the removal of a weighted cuff (Gregory \& Ross, 1967) or immersion in water (Ross \& Rejman, 1973; Synodinos \& Ross, 1979). However, discrimination recovers almost to its normal value when time is allowed for adaptation to the change in arm weight. Discrimination also deteriorates when the arm weight is unchanged but the range of weights is changed. For example, prior lifting of heavy weights temporarily raises the threshold for discrimination between lighter weights (Holway, Goldring, \& Zigler, 1938), and a varying standard yields a higher threshold than a constant standard (Woodrow, 1933). Ross and Gregory (1970) found a similar result for purely cognitive factors: the size-weight illusion raised the threshold slightly above optimum for objects that were apparently too heavy or too light for their size. The reported changes in threshold due to maladaptation are usually not greater than a factor of 2 . Holway et al. (1938) reported changes up to a factor of 6 , but they used an adjustment method that yielded thresholds about 10 times higher than those normally found by the usual methods, so their results should be treated with caution.

Both of the general rules discussed above have some bearing on the microgravity phase of parabolic flight. One can predict a deterioration in discrimination on at least two grounds: (1) there is a loss of normal weight cues, leaving only the inertial cues to mass, and (2) the subject is maladapted to the sudden loss of body weight and object weight, and has insufficient time to adapt. On either ground, one might predict a raising of the threshold by a factor of up to 2 , and the combined effect might be even greater.

The second rule also has a bearing on the macrogravity phase of parabolic flight. One can again predict a raising of the threshold due to maladaptation, as in the human centrifuge. However, since the weight cues are not lost, the deterioration should be less than it would be under microgravity.

A preliminary experiment conducted during the microgravity phase of parabolic flight suggested that the DL for mass discrimination was, indeed, about twice as large under $0 \mathrm{G}$ as under $1 \mathrm{G}$. Nine subjects were tested with a set of weighted balls whose mass ranged from 48 to $72 \mathrm{~g}$. The subjects, given a series of pairs of balls, shook each pair and then decided which of the two was heavier. The balls were attached to a box by long strings. A photograph of the apparatus in use, with the balls floating under micro-

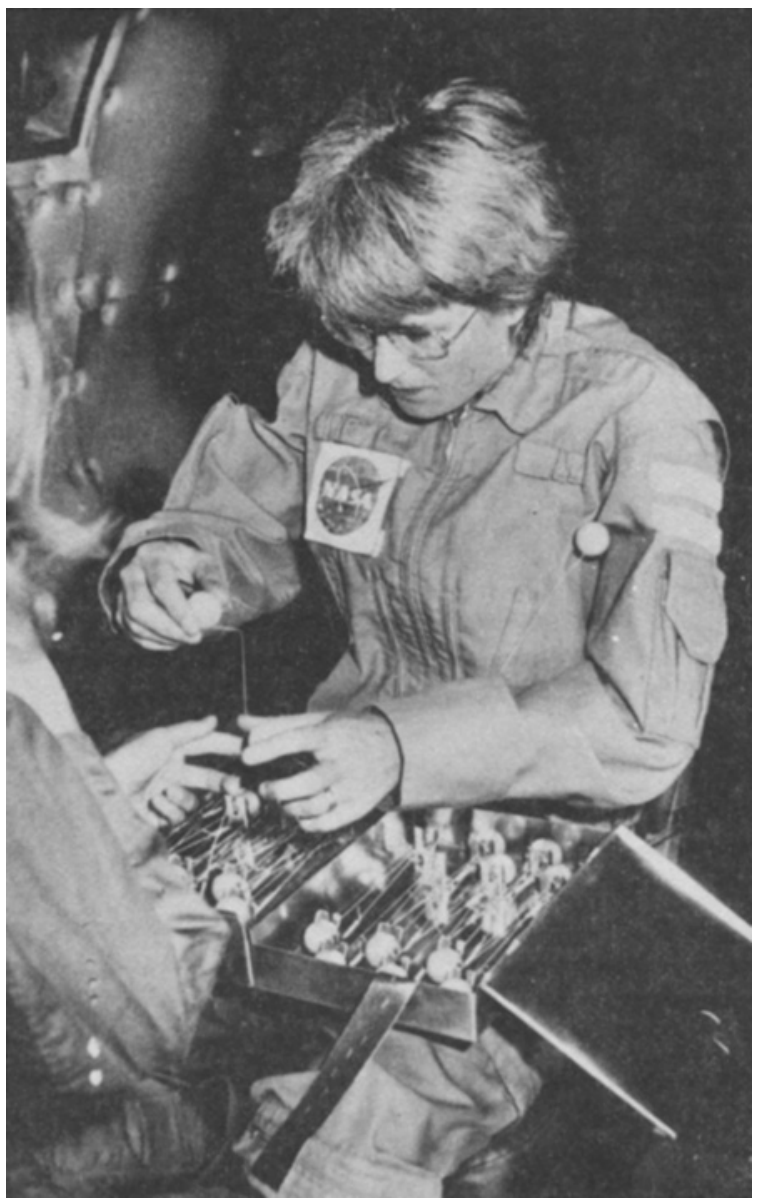

Figure 4. Preliminary version of the mass-discrimination apparatus in use during parabolic flight.

gravity, is shown in Figure 4. The subjects made significantly more errors in flight than on the ground. The DL, calculated from the group data, was $9.95 \mathrm{~g}$ in flight and $5.26 \mathrm{~g}$ on the ground. The mean mass of the balls lifted was $55.8 \mathrm{~g}$, giving Weber fractions of .18 under $0 \mathrm{G}$ and .09 under $1 \mathrm{G}$. Unfortunately, the range of masses carried in the experiment turned out to be far too large, with the result that relatively few errors were made and the estimated thresholds were unreliable. The experiment was therefore repeated with a more suitable range of masses, and the effect of macrogravity was also investigated.

\section{Method}

The subjects were seven men and two women, aged between 21 and 41 years (mean, 27 years). All were right-handed. One subject had experienced over 50 parabolic flights, but the remainder were on their first or second flight. They were NASA employees, who had volunteered to participate in experimental flights. Two subjects were premedicated with scopolamine/dextroamphetamine as part of another experiment.

The apparatus was identical to a model designed to be flown in the first Spacelab mission and is described in greater detail elsewhere (Ross, 1981a). It consisted of an aluminum box which un- 
folded to form two trays, each measuring $300 \times 200 \times 70 \mathrm{~mm}$. The box contained 24 balls, 12 in each tray. The balls were $30 \mathrm{~mm}$ in diameter and rested in holes in a metal plate, secured in place by elastic straps. The balls were constructed of epoxy resin, with a central annulus of lead. The spacing of the lead annulus was varied, so that the balls had equal moments of inertia (4.0-4.1 $\times$ $\left.10^{-6} \mathrm{~kg}^{2}\right)$ despite variations in mass. The mass varied from 50 to $64 \mathrm{~g}$, in $2-\mathrm{g}$ steps. There were 10 balls of $50 \mathrm{~g}$ and 2 each of the other values. They were labeled randomly with letters of the alphabet and arranged alphabetically in the trays.

The subjects were trained and given a pretest at Ellington Air Force Base about $1 \mathrm{~h}$ before takeoff. The experimenter demonstrated how to pick up a ball from under its restraining strap, shake it from the wrist two or three times while holding it loosely in the hand, and then replace it in its hole under the strap. The experimenter then called out a pair of letters. The subject shook and replaced each of these in the prescribed order and reported which felt heavier. The subject was given six practice trials before the pretest. The test consisted of 16 pairs. These pairs represented four step intervals $(2,4,6$, and $8 \mathrm{~g})$ repeated four times, twice with the first ball heavier and twice with the second. For 10 of the trials, the lighter ball was $50 \mathrm{~g}$, and for the other trials, it was 52 , 54,56 , or $58 \mathrm{~g}$. Lists were constructed so that no letter pairs were repeated, and so that all lists were equivalent in difficulty. The mean mass in each list was $54.4 \mathrm{~g}$. (The purpose of this unconventional design was that subjects could test themselves in Spacelab and other situations, without being able to learn that certain balls were always heavier. The experimenter administered the test on this occasion to save time. It is intended to compare these results with a similar, but self-administered, test in Spacelab.)

Five lists were prepared, and each subject was given a different order of lists for the five different tests (two ground tests and three flight tests). The subject and the experimenter sat side by side at a table, with the box open on the table in front of the subject. The experimenter checked off the subject's responses on the list. The subject worked at the rate of about $10 \mathrm{sec}$ per comparison. A complete test lasted about $21 / 2-3 \mathrm{~min}$.

The subjects were tested again during the flight. The subject and the experimenter sat side by side, strapped in chairs at the back of the aircraft. The box was strapped open on the subject's lap. The experimenter called out the pairs of balls to him and recorded his responses on the list. The subject normally made two comparisons during the 1.8-G early phase of the parabola, then two during the $0-G$ phase, then two during the $1-G$ interparabola phase. (The late macrogravity phase was too brief and variable to be of use for testing.) This sequence was repeated over 8-10 parabolas, until all trials were completed. When time permitted, some subjects then made magnitude estimates throughout one or two parabolas, as described in the discussion of Experiment 1. The tests were conducted over 3 days of flights.

The posttest was carried out in the same way as the pretest, within 30 min of landing.

\section{Results and Discussion}

The mean number of errors for the five tests is shown in Table 2. The means ranged from 4.0 to 6.1

Table 2

Mean Number of Discrimination Errors (of 16) and the Corresponding DLs and Weber Fractions for the Ground and Flight Tests

\begin{tabular}{lrrrrrr}
\hline & \multicolumn{2}{c}{ Ground (1-G) } & & \multicolumn{3}{c}{ Flight } \\
\cline { 2 - 3 } \cline { 5 - 6 } & Pretest & Posttest & & $1-G$ & $1.8-G$ & $0-G$ \\
\hline Errors & 4.78 & 4.00 & & 4.11 & 5.56 & 6.11 \\
DL (in Grams) & 6.29 & 4.87 & & 4.62 & 8.52 & 9.90 \\
Weber Fraction & .12 & .09 & .09 & .16 & .18 \\
\hline
\end{tabular}
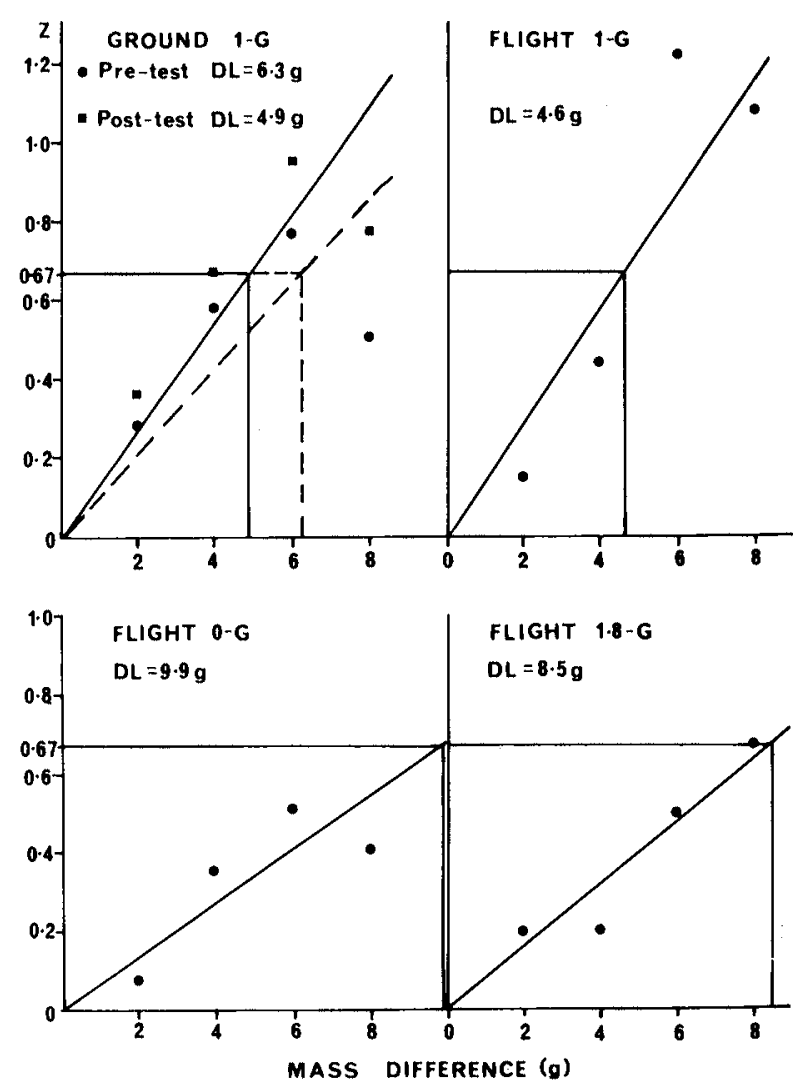

Figure 5. Differential thresholds derived from group data at various $G$ levels in flight and on the ground.

$(25 \%$ to $38 \%)$. The effect of the different conditions just failed to reach significance on an ANOVA $[F(4,32)$ $=2.42, p=.068]$. However, since the directions of the differences are highly predictable, it is reasonable to use one-tailed tests. On this basis, 0-G performance was worse than that at $1 \mathrm{G}$ in flight $[\mathrm{F}(1,32)=5.79$, $\mathrm{p}=.011]$ and than that at $1 \mathrm{G}$ postflight $[\mathrm{F}(1,32)=$ $6.38, p=.008$ ]. Performance at $1.8 \mathrm{G}$ was also worse than at $1 \mathrm{G}$ in flight $[\mathrm{F}(1,32)=3.03, \mathrm{p}=.046]$ and postflight $[F(1,32)=3.50, p=.036]$. No other comparisons were significant.

The DLs were calculated for the group data, as there were insufficient data for individual analysis. The percentage of correct judgments at each step interval was converted to a $\mathrm{z}$ score, and a straight line was fitted through the origin and the mean of the $z$ scores at the mean interval. The DL was taken as the interval corresponding to $75 \%$ correct $(z=.67)$. The DLs are listed in Table 2, and the data points are shown graphically in Figure 5.

The fit of the data points to the lines is ragged, partly because of shortage of data, but mainly because the lighter ball was not always $50 \mathrm{~g}$ but sometimes heavier. According to Weber's law, this has the effect of depressing performance for pairs taken from higher up the range. This effect is mainly evident for 
the 8-g interval, in which the heavier balls were used relatively too often (in a successful attempt to prevent the subjects from learning that certain balls were always the heavier of a pair). These irregularities are taken into account in calculating the Weber fraction, and do not contribute to differences in performance between conditions.

A Weber fraction of .09 to .12 on the ground may seem high in relation to the value of .02 that is quoted in many textbooks (probably following Boring, Langfeld, \& Weld, 1939). However, the more normal range seems to be about .05 to .12 (Woodworth \& Schlosberg, 1961, p. 224). Our results appear to be quite normal for unpracticed subjects using light weights. The high pretest Weber fraction was almost certainly due to lack of practice. The posttest and $0-G$ values were almost identical to those of the pilot study.

The results were much as expected. Performance at $1 \mathrm{G}$ in flight was the same as at $1 \mathrm{G}$ postflight. This is reassuring, because it means that poor performance under macrogravity and microgravity is not due simply to the anxieties and distractions of the flight. The DL under $0 \mathrm{G}$ was about twice as high as that at $1 \mathrm{G}$-the same finding as in the preliminary experiment. The DL at $1.8 \mathrm{G}$ was slightly lower than at $0 \mathrm{G}$, though not significantly so. This is interesting, because it suggests that there is nothing unique about microgravity: a change to macrogravity can be almost as disruptive. The loss of weight cues in microgravity is probably of some importance, but the lack of time for adaptation is probably of greater importance. It is instructive to compare macrogravity performance in parabolic flight with that in the human centrifuge (Ross, 1981b): in the latter case, the Weber fraction under $2 \mathrm{G}$ rose by a factor of about 1.3 , compared with about 1.8 in flight. The smaller effect in the centrifuge was probably due to the fact that the test lasted for about $5 \mathrm{~min}$, thus allowing some opportunity for adaptation.

The conditions of prolonged spaceflight are much less distracting than those of parabolic flight, and they allow time for adaptation. Performance should therefore be relatively good in spaceflight. It has, for example, been reported that manual performance slows down slightly during the $0-G$ phase of parabolic flight (Wade, Note 4); but Jackson (Note 5) found no degradation in eye-hand coordination during the Skylab 4 mission. The same may well be true of mass discrimination.

It is interesting that the results from parabolic flight suggest that microgravity is slightly more deleterious than macrogravity. If the difference is genuine, there might be several reasons for it. It may be that adaptation is asymmetrical, the nervous system taking longer to adapt to a reduction in stimulus intensity than to an increase. Or it may be, as suggested earlier, that the total loss of weight cues is a deleterious factor which must be added to that of maladaptation. Alternatively, the loss of weight cues might be regarded as an extreme example of the breakdown of Weber's law for very light masses. Unfortunately, there appears to be no satisfactory experimental literature on this subject. Holway and Hurvich (1937) found large Weber fractions over the whole range and huge increases for very light masses, but their adjustment method means that the results are confounded with the question of the rate of adaptation. Oberlin (1936) used the method of constant stimuli and found roughly constant Weber fractions, ranging from .04 to .07 for masses between 50 and $600 \mathrm{~g}$, but a value of .12 at $25 \mathrm{~g}$. Ross (1964), also using the constant method, found Weber fractions of about .07 at 75 and $200 \mathrm{~g}$, but .14 at $10 \mathrm{~g}$. These isolated examples suggest that the Weber fraction may rise by a factor of about 2 for very light masses (well below $50 \mathrm{~g}$ ) in comparison with middle range masses. However, a systematic investigation over the lower range would be necessary to establish this. Whatever the outcome of such an investigation, one should not readily assume that loss of weight due to loss of gravity is equivalent to loss of weight due to loss of mass.

So far, we have assumed that performance in spaceflight will be either worse than at $1 \mathrm{G}$ or no different. There remains the possibility that mass discrimination might actually be better during prolonged weightless conditions. This might be the case if earth's gravity contributes in some way to background noise and tends to mask inertial cues (which may be inherently more accurate than weight cues). It might have such an effect because the acceleration of gravity is high in comparison with that produced by normal hand movements or because it makes the arm heavy. Fechner $(1860 / 1966)$ suggested that the weight of the arm contributes in a small way to the denominator of the Weber fraction, causing the raising of the fraction for light weights. We know that the brain compensates quite well for changes in arm weight (Gregory \& Ross, 1967), but perhaps not entirely. Possibly, then, discrimination might improve in the absence of gravity, although the mass of the arm would still contribute to inertial stimulation.

Whatever the outcome in space, we might expect an aftereffect of poor discrimination on return to earth. It is known that postural equilibrium is disturbed for some days postflight (Homick, Reschke, \& Miller, 1977), and that the body and other objects feel too heavy (Gazenko et al., Note 2). It would not be surprising if weight discrimination were also affected.

\section{REFERENCE NOTES}

1. Crawford, S. M., \& Kama, W. N. Remote handling of mass (ASD TR 61-627). Wright-Patterson Air Force Base, Ohio, 1961.

2. Gazenko, O. G., Genin, A. M., \& Egorov, A. D. Summary of medical investigations carried out in the USSR manned space 
missions. Paper presented at the 6th International Symposium on Man in Space, Bonn, 1980.

3. Rees, D. W., \& Copeland, N. K. Discrimination of differences in mass of weightless objects (WADD Tech. Rep. 60-601). WrightPatterson Air Force Base, Ohio, 1960.

4. Wade, J. E. Psychomotor performance under conditions of weightlessness (MRL-TDR-62-73). Wright-Patterson Air Force Base, Ohio, 1962.

5. Jackson, K. Motor sensory performance in Skylab 4. In M. Anderson (Ed.), BIOSPEX: Biological space experiments (Tech. Mem. 58217). Washington, D.C: NASA, 1979. (Abstract)

\section{REFERENCES}

Boring, E. G., Langfeld, H. S., \& Weld, H. P. Introduction to psychology. New York: Wiley, 1939.

Fechner, G. T. [Elements of psychophysics] (H. E. Adler, D. H. Howes, \& E. G. Boring, trans.). New York: Holt, Rinehart \& Winston, 1966. (Originally published, 1860.)

Fischel, H. Transformationserscheinungen bei Gewichtshebungen. Zeitschrift für Psychologie, 1926, 98, 342-365.

Gandevia, S. C., \& McCloskey, D. I. Sensations of heaviness. Brain, 1977, 100, 345-354.

Gregory, R. L., \& Ross, H. E. Arm weight, adaptation, and weight discrimination. Perceptual and Motor Skills, 1967, 24, $1127-1130$.

Holway, A. H., Golmning, L. E., \& Zigler, M. J. On the discrimination of minimal differences in weight: IV. Kinesthetic adaptation for exposure intensity as variant. Journal of Experimental Psychology, 1938, 23, 536-544.

Holway, A. H., \& Hurvich, L. M. On the discrimination of minimal differences in weight: I. A theory of differential sensitivity. Journal of Psychology, 1937, 4, 309-332.

Homick, J. L., Reschke, M. F., \& Miller, E. F. The effects of prolonged exposure to weightlessness on postural equilibrium. In R. S. Johnston \& L. E. Dietlein (Eds.), Biomedical results from Skylab (NASA). Washington, D.C: U.S. Government Printing Office, 1977.

McCloskey, D. I., Ebeling, P., \& Goodwin, G. M. Estimation of weights and tensions and apparent involvement of a "sense of effort." Experimental Neurology, 1974, 42, 220-232.

ObERLIN, K. W. Variation in intensive sensitivity to lifted weights. Journal of Experimental Psychology, 1936, 19, 438-455.
Poulton, E. C. Models for biases in judging sensory magnitude. Psychological Bulletin, 1979, 86, 777-803.

Ross, H. E. Constant errors in weight judgements as a function of the size of the differential threshold. British Journal of Psychology, 1964, 55, 133-141.

Ross, H. E. When is a weight not illusory? Quarterly Journal of Experimental Psychology, 1969, 21, 346-355.

Ross, H. E. Mass-discrimination during weightlessness: Plans for a Spacelab experiment. In $21^{\circ}$ Convegno Internazionale Scientifico Sullo Spazio. Rome: Rassegna Internazionale Elettronica Nucleare Ed Aerospaziale, 1981. (a)

Ross, H. E. How important are changes in body weight for mass perception? Acta Astronautica, 1981, 8, 1051-1058. (b)

Ross, H. E., \& Bischof, K. Wundt's views on sensations of innervation: A reevaluation. Perception, 1981, 10, 319-329.

Ross, H. E., \& Gregory, R. L. Weight illusions and weight discrimination-A revised hypothesis. Quarterly Journal of Experimental Psychology, 1970, 22, 318-328.

Ross, H. E., \& REJMan, M. H. Weight discrimination in water. In N. C. Flemming (Ed.), Science diving international. Andover, Hants: Standard Press (British Sub Aqua Club), 1973.

Ross, H. E., Rejman, M. H., \& Lennie, P. Adaptation to weight transformation in water. Ergonomics, 1972, 15, 387-397.

Stevens, S. S., \& Galanter, E. H. Ratio scales and category scales for a dozen perceptual continua. Journal of Experimental Psychology, 1957, 54, 377-411.

Srnodinos, N., \& Ross, H. E. Water temperature and weight discrimination. Ergonomics, 1979, 22, 1083-1093.

Torrey, C. C. The distance-weight effect: An exploratory study in weight perception (Doctoral dissertation, Cornell University, 1963). Dissertation Abstracts, 1964, 24, 3858. (University Microfilms No. 64-3672)

WEBER, E. H. [The sense of touch] (H. E. Ross \& D. J. Murray, trans.). London: Academic Press, 1978. (Originally published, 1834.)

Woopkow, H. Weight-discrimination with a varying standard. American Journal of Psychology, 1933, 45, 391-416.

Woodworth, R. S., \& Schlosberg, H. Experimental psychology. London: Methuen, 1961.

(Manuscript received December 29, 1980; revision accepted for publication February 5, 1982.) 